\title{
Design-Thinking and UCD Combination for Designing Effective Time Management Assistant Mobile App
}

\author{
Ashana Liyanage \\ Dept. of. IT \\ Sri Lanka Institute of \\ Information \\ Technology \\ New Kandy Road, \\ Malabe, Sri Lanka \\ shinirmani1997@gmail.com
}

\author{
Samanthi Siriwardana \\ Dept. of IT \\ Sri Lanka Institute of \\ Information \\ Technology \\ New Kandy Road, \\ Malabe, Sri Lanka \\ samanthi.s@sliit.lk
}

\author{
Malithi Mithsara \\ Dept. of IT \\ Sri Lanka Institute of \\ Information \\ Technology \\ New Kandy Road, \\ Malabe, Sri Lanka \\ malithi.mith@gmail.com
}

\author{
Shyam Reyal \\ Dept. of IT \\ Sri Lanka Institute \\ of Information \\ Technology \\ New Kandy Road, \\ Malabe, Sri Lanka \\ shyam.r@sliit.lk
}

\begin{abstract}
This project presents the high-fidelity prototypes of a smart digital personal assistant for university students in the form of a mobile app, using a combination of User-Centered Interaction Design and Design Thinking methodologies. The end goal is to have an effective and user-friendly set of interfaces that display the right amount of information to the user without missing key details while not showing too much information such that the user becomes overwhelmed. The target audience benefitting from this work are university students who are reactive in nature and do not possess the required perseverance to complete the tedious initial setting up process of a Time Management Tool (TMT). The study was conducted involving university students using surveys, interviews and focus groups. This sheds light on key time management issues faced by university students and paves the way for future research and development of TMTs.
\end{abstract}

Keywords - User Centered Interaction Design (UCID), Design Thinking, Time Management, Human Computer Interaction, Time Management Tool (TMT)

DOI: 10.37789/ROCHI.2021.1.1.17

\section{INTRODUCTION}

It is a known fact that university students struggle to manage time and prioritize between studies and personal commitments. Although an expanding number of computer-based Time Management Tools (TMTs) have been developed to address this problem, not much success has been achieved as the initial setting up, data entry and configuration is tedious.

TMT's such as Trello [16], TodoIst [17], and Google Calendar [18] are popular among students. However, one single tool may not be enough forcing a person to use multiple tools in combination. Although several integration mechanisms and multi-functional TMTs are available, they are tedious to configure. Existing TMTs are only effective to people who are proactive and organized, who will actively add reminders, events, tasks, assignments, projects to be completed. However, these must be entered manually, and does not sit well with reactive or unorganized users - who will be satisfied upon seeing their work being completed, yet do not have the perseverance and discipline to complete the initial configuration and data entry.
Existing studies largely concern the user experiences of those individual users and group users for a single tool. Yet, few studies focus on the user experiences of those who use multiple TMTs, $[1]$ and none catering for the needs and circumstances of university students were found. This paper presents highfidelity prototypes of a TMT mobile app called ScheduleME, be designed based on students' needs and requirements. The students only need entering very few details into ScheduleME, and the relevant and right amount of information will be presented to the user in the most user-friendly way possible.

\section{BACKGROUND AND LITERATURE REVIEW}

This section provides a comprehensive background study into Time Management, User Centered Design, Design Thinking, review of existing TMTs and other research carried out on time management.

\section{Time Management}

Time management is a very generally defined concept in literature. Numerous terms are used in the literature to refer to time management - such as task management and project management. However, all these terms belong to different fields. The term time management was originally introduced in the 1950s only to deal with job-related problems. [3]. In the early 1990s, time management was broken down into a group of related aptitudes that can be evaluated and quantified separately. The nine subscales are "Sense of Purpose, Structured Routine, Present Orientation, Effective Organization, Persistence, Setting Goals and Priorities, Mechanics, Perceived Control of Time, and Preference for Organization" [3]. Many psychological symptoms are proved to correlate with lacking time management such as anxiety, depression, low life, and work satisfaction [3]. Conversely, effective time management is a significant element that induces success in various domains [6]. More studies were carried out concerning students' lives later and many studies found that efficient time management help improves academic grade and build more productive study habits [3].

\section{User Centered Design}

User-centered design (UCD) was developed in the 1980s at Donald Norman's Research Laboratory at the University of California, San Diego [2,4]. However, the potential for UCD was recognized more than two decades ago by Nickerson, who suggested that "people-centered computers" were a necessity and trend in the future. UCD is a methodology and philosophy 
that emphasizes user needs and user involvement throughout the design process. It can combine with agility, waterfall, and other methods. It follows a flexible and iterative design lifecycle [2] which covers the planning, design, implementation, and testing phases.

Another feature of UCD is its ease of use. The user experience is more valuable than usability principles during the process and decision-making. Macintosh UI designers [2,3] agree that users do not always prefer the most effective and efficient design, and some empirical results show that users sometimes prefer poorly designed UI more. Therefore, all user experience considerations should be made according to and based on the users' experience.

\section{Design Thinking Methodology}

Design Thinking is a problem-solving and project-management approach that adopts the techniques used by designers, the way they work, and how they approach problem-solving to re-think different models in diverse fields (business, health, etc.) It also modifies or creates new techniques that are suitable for users while utilizing available technology. The number of industries in which Design Thinking is used has grown tremendously in recent years [7].

A generally accepted definition of design thinking has yet to emerge [9], and even the term itself is a subject of controversy among its practitioners and advocates [8]. The nomenclature of "design thinking" first appears prominently in a book of that title authored by Peter Rowe (1987), a professor of architecture and urban planning at Harvard's School of Design. A review of that publication's contents reveals the usage of the term primarily oriented to architectural design that does not capture its current meaning as practiced in the business environment [6].

\section{Existing Time Management Tools}

This section reviews some existing TMTs in popular use today, classified into two groups based on the intended user. One serves individual use, and the other group serves group use.

\section{Time Tracking Tools - RescuTime, Toggle}

These are used to track time used on each task and generate visualizations and reports to analyze the user's time distribution. Consequently, it helps the users understand their working time usage and improve productivity. A major shortcoming of the time tracking tool is that it is prone to place unnecessary pressure on the users by visualizing the time of users engaging in distractions in a punishing manner [10].

\section{Note Taking - Remember the Milk, Todolst, MinimaList,} Evernote, Doris, Clear

These allow users to note down and organize tasks in an easy and timely manner. Apart from making a to-do list and setting goals, a task reminder is another commonly used function of these TMTs. They are useful for reminding appointments and to-dos and various time-based information such as colleagues' birthdays [4]. Inevitably, there are some limitations with these tools, such as prioritizing and expensiveness [5].

Scheduling -DayViewer, Week Plan, Weekis, GQueues These aid in the management of schedules and are often presented in the form of a calendar or a semi-structured note. Activities appear as blocks on the calendar and maybe labeled in a variety of ways to distinguish between distinct tasks or projects. As they take a lot of time and effort to set up, input, and maintain, these technologies are more commonly utilized by groups. They are less adaptable and accessible than paper planners. Present planning and scheduling systems are not commonly used because they need too much input and work to maintain. [8].

\section{Group Collaboration - Trello, Asana, Slack, Timely}

TMTs for group use mostly aim for business use as they offer more complex but powerful features such as real-time changes and third-party integrations. Though they have more functions for team collaboration, users who only need functions for the individual purpose may find it ineffective to use. The common issues include data security, steep learning curve, and price [12]. Most of the tools offer full features at a price, which in the context of university students is an inconvenience.

Integrated Platforms - Google Keep, Office365 Tasks, Google Calendar, Office365 Calendar, Google Drive, One Drive, SharePoint

Google and Microsoft Office are two well-developed integrated platforms, which have multiple applications providing different functions such as calendars and to-do lists. the users can access all the functions with one account and the functions can synchronize with each other automatically [18]. However, a significant concern of using these tools is data privacy. The dilemma exists between convenience and privacy. The more data you give to the service providers, the better they understand your needs, thereby providing a better-designed service. Also, these tools are developed for general working purposes and do not contain any time management techniques [11].

\section{Other Research Carried on Time Management}

\section{Task Switching and Interruptions}

Microsoft Research [19] investigated how people interleave multiple tasks amidst interruptions. They chose a group of 11 experienced Microsoft Windows users, ranging in age from 25 to 50 years working as stockbrokers, web designers, software developers, network administrators, and computer science professors. They were required to multitask on more than three projects and recorded the average difficulty of switching to another task by using Microsoft Excel. After gathering and analyzing diary data from the participants, they evaluated designs of prototype task-management tools that were generated by issues found in the study. The participants' most prevalent design suggestions focused on the development of new reminder tools, such as the potential value of cross-application project and to-do list tracking.

\section{Interruptive Notification in Task Management}

Celeste et al [20] investigate ways to support task management in the desktop environment with interruptive notifications. They performed two user studies with $\mathrm{KDH}$ open-source software users and developers to learn more about how they deal with interruptive notifications. They found that interruptive notifications support a user's task management activities and are essential for a worker-friendly computing environment. Certain notifications were found to enhance multitasking, task prioritization, and task disruption management. 


\section{Work Fragmentation and Task Management Practices}

Franssila et al. [21, 22] conducted a study on task management strategies and work fragmentation experience among knowledge workers. They performed an online survey to assess the effectiveness and efficiency of task management and work fragmentation. The findings of this study reveal a negative work fragmentation experience and a loss of control over task performance. On the other hand, this work fragmentation also led to mental stress. They also analyzed the association between email intensity in work time spending and subjectively experienced quality of work performance. The study was conducted over five working days in an office and all participants used personal computers with Windows. They recorded the computing activity of all users by using "Manic Time" tracking software and MS Outlook. They discovered that intensity of email use does not impair subjectively experienced productivity.

It can be seen that the common drawbacks of TMTs include "portability," "being readily accessible," "visual salience in the workplace," "fluidity of visual structure," and "scarring" [5]. There are also issues regarding data security in general.

\section{METHODOLOGY}

With university students, the design should be simple and straightforward such that it won't take an unnecessary amount of time to learn and use. UCD was selected as the optimum choice to carry out the project to ensure the outcomes are simple and straightforward to users - as the products are completely designed upon the user's needs from requirements gathering to the acceptance testing. To obtain the widest and deepest understanding of the university students, this research employs a pragmatic approach which contains both qualitative and quantitative methods which complement each other.

The main objective is to present high-fidelity prototypes of an effective time management and task prioritizing mobile app that is effortless to use, that does not require unnecessary amounts of time to learn, use, and meet the needs of university students. User Centered Design and Design Thinking methodologies are utilized to implement the research.

The main research objective is realized via four sub-objectives - mapped to the appropriate phase-in Design Thinking process in parentheses:

1. To analyze and understand students' work and needs using data gathering, data analysis and model building tasks (Empathize and Define).

2. To create interaction design concepts to create viable solutions rapidly to meet the specified requirements and UX goals (Ideate)

3. To realize design alternatives using prototypes (Prototype)

4. To verify and refine interaction design assessing the outcomes of the evaluation against the users' context and requirements to check how well a design is performing (Test).

\section{Empathize}

The first stage of the design thinking methodology is Empathize. Gaining an empathic understanding of the matter that ought to be solved is that the main thing tried at this stage. This involves understanding the user and their needs by being in their position. Methods used for this stage are Online Surveys and Interviews. Researchers collect data from users' working context as the initial task emphasizes the authenticity of the data. Data collected should be raw and genuine so the reliability of the following analysis can be maximized [15].

An online survey was distributed among students in Sri Lanka Institute of Information Technology (SLIIT) receiving 101 responses covering various demographical backgrounds. In this section, the design, the implementation, and the findings of the survey are illustrated.

The survey objectives were to identify (a) what are the time management and task management issues faced by a university student (b) their utilization level of the time management tasks, (c) the familiarity level of the TMTs, (d) the frequency of using the TMTs and (e) preferred methods for boosting productivity.

Surveys and questionnaires are insufficient to capture some user requirements, such as emotions and beliefs [13]. To balance this, qualitative methods are used to capture deeper insights into the users' behavioral and psychological facts. Thus, interviews were used to gather qualitative data.

Even though the questions asked in each interview were the same, the answers received were diverse.

The main questions asked in the survey and interviews were:

- What are the common time management problems you have?

- Do you use time management tools?

- If yes, what time management app(s) do you use?

- What are the limitations and shortcomings you see in those tools?

- How often do you use the time management tool or how often do you enter data to the time management tools?

- Do you forget to enter data/tasks to those time management apps daily or constantly?

- When it comes to data entering, how often would you like to enter data to the time management tool you use? (To be productive and get tasks done)

- Assume an assignment with a big workload is uploaded to the Learning Management System (LMS) of the university. The deadline is two weeks away. What would you do in the above scenario?

- Have you ever missed something important that was posted on LMS because you forgot to $\log$ in to that relevant module and check?

- How do you usually find out about new assignments, lectures, notices etc. posted on LMS?

- If a notification is sent to you when a new notice/assignment is posted to LMS how soon would you like to receive it and know about the new update posted on LMS 
- What is your most active time of the day based on the following scenario? "Assume you have an exam in one week. What time do you think you can study and focus better, be most productive"?
- How well do you keep the balance between your academic activities (lectures, assignments etc.) and personal chores and allocate time to them?

- How many hours do you usually sleep per day?

How do you usually spend your spare time?

\begin{tabular}{|l|l|l|l|}
\hline Method & Participant Demographic Information & $\begin{array}{l}\text { Question } \\
\text { type }\end{array}$ & $\begin{array}{l}\text { Qualitative/ } \\
\text { Quantitative }\end{array}$ \\
\hline $\begin{array}{l}\text { Online } \\
\text { Survey }\end{array}$ & $\begin{array}{l}\text { 101 responses. More than 90\% of participants were 1 } 1^{\text {st }} \text { and 4 } 4^{\text {th }} \text { year students from } \\
\text { SLIIT. Participants were in different age groups, different hometowns, and } \\
\text { following different degree programs. Participants were between 19-26 years old. }\end{array}$ & $\begin{array}{l}\text { Close ended } \\
\text { questions }\end{array}$ & Quantitative data \\
\hline Interviews & $\begin{array}{l}5 \text { participants were interviewed. We interviewed one student from each year } \\
\text { across all 4 years at SLIIT and 1 interview with a student from another university. } \\
\text { They were from different age groups and following different degree programs. }\end{array}$ & $\begin{array}{l}\text { Open ended } \\
\text { questions }\end{array}$ & Qualitative data \\
\hline
\end{tabular}

Table 1. Method used for Empathize.

\section{Define}

The second stage is Define. In this stage, the researcher has analyzed observations and arranged them to define the core problems that have been identified in the previous stage. According to the qualitative and quantitative data, I gathered from the empathize stage, the main identified core problems are,

1) Students have multiple time management problems. the most common ones are prioritization of tasks. others are difficult to multitask, procrastination, starting the day late, perfectionism, ineffective scheduling, failing to manage numerous distractions., skipping on breaks etc.

2) Existing time management tools have shortcomings such as having to enter all the details manually, too much information is shown in the app, less information is shown (key details are missed), less user-friendly, hard to prioritize the tasks, does. not make reminders. etc.

3) Students often forget to enter details and update the time management apps daily and consistently.

4) Most of the students only like to enter data once a week.

5) Half of the students are reactive students - they respond to events after they have occurred. On the other hand, a proactive student focuses on eliminating problems before they happen.

6) Most of the students have missed key details posted on LMS multiple times.

7) More than half of the students find recent updates posted on LMS by social media groups such as WhatsApp, from batchmates and not by logging into LMS.

8) Many students struggle with managing time between personal commitments and academic deadlines

\section{Ideate}

Ideate is the third stage of the design thinking process. This stage aims to create viable solutions rapidly to meet the specified requirements and UX goals. It will be helpful to find solutions to problems that are identified in the define stage and find alternative ways to display the problem.

The methods and techniques used in this stage are a brainstorming and focus group. Ten university students participated, and nine were from SLIIT. Everyone had to speak and share their ideas about the topic. Everyone was allowed to speak freely and without fear of judgment. In the beginning, the research and the app intended to develop (ScheduleME) were briefly described to the participants. Some of the main questions asked and the ideas collected are mentioned below

Question 1 - what kind of techniques do you use to deal with time management problems and be productive?

Ideas collected in the brainstorming session-

- Make to-do lists and set reminders.

- Reward yourself after finishing a task.

- Divide a big task into small tasks and make a to-do list.

- Focus on a few things at a time.

- Focus on high-value activities first.

- Just start to do the task without procrastinating.

- Be consistent

- Clean your place or desk, making an appropriate environment to do the tasks.

- $\quad$ Practice the 10-minute rule.

- Evaluate how you are spending your time.

- Limit distractions.

- Get plenty of sleep, eat a healthy diet, and exercise regularly.

- Take a break when needed.

Question 2 - According to survey responses and interviews a majority of students used time management apps. But there are several shortcomings of those apps such as having to enter all 
the details manually, being hard to prioritize the tasks, being less user-friendly, etc. What features do you think should be added or improved on those apps?

Ideas collected in the brainstorming session -

- The user is only required to enter data to the TMT at most once a week.

- Prioritize daily task list automatically.

- Displays only the relevant information to the user.

- Can view both completed and uncompleted tasks.

- Can view the upcoming assignments, meetings, exams in calendar user interface.

- Suggest tasks to the user according to their preferences.

- Send notifications when something is posted on LMS

- Can view both finished tasks/assignments and upcoming tasks/assignments.

- Can view the time user spent on social media and other apps.

- Display the time needed to allocate for a certain task automatically.

- Display the completed progress of the assignments that the user is currently being doing.

Responses collected for the above from participants were applied and when designing TMT prototypes.

The diverse behavior of students was also discussed. Some can concentrate and work better in the mornings. Some perform better at night. Users should carry out work at their most active and favored times. If a student can study and work better at midnight, he/she should do the high-priority tasks at that hour. It won't be as effective if he/she is expected to do this first thing in the morning. Therefore, high-priority activities should be allocated to the most convenient time. Then there are other responsibilities, such as personal chores, that take up the rest of the day. The student will be able to complete academic assignments and personal responsibilities more efficiently.

At the end of the focus group, the most important distinction between students was discussed. There are reactive and proactive students. According to the survey and interviews, half of the students are reactive students. We discussed a few techniques to how to be a prepared and proactive student without rushing at the last moment to complete a deadline. Some of them are making a timetable and allocating an abbreviated time each day to do a task etc.

\section{Prototype}

The fourth stage of design thinking methodology is involved in this third sub-component. A prototype is an early sample, model, or release of a product built to test a concept or process. This is an experimental phase. There are two types of prototypes called low fidelity prototype and high-fidelity prototype.

Low-fidelity (lo-fi) prototyping is a fast and uncomplicated way to translate high-level design concepts into real, tangible, and testable artifacts. The vital role of low-fidelity prototypes is to check and test the functionality of the product rather than the physical appearance.
Low fidelity prototypes were designed according to the requirements gathered from the university students in the previous stages. (Interviews, online-survey, focus group, and brainstorming session) using PowerPoint.

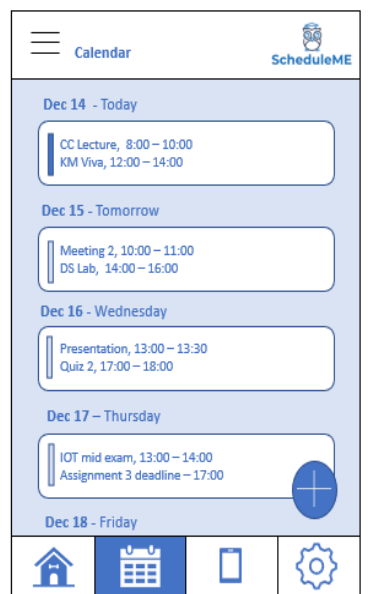

Figure 1. low-fi list



Figure 2. low-fi calendar
A new survey was created to get feedback for low-fidelity prototypes and twenty-three responses were there as an output for the survey. The questions asked were about the userfriendliness in the prototypes, limitations, and shortcomings in the prototypes, etc. According to the feedback, $76.2 \%$ of students have said they would prefer to see their daily schedules as in the calendar list view (Figure 1), rather than the calendar view (Figure 2).

High Fidelity prototypes were built for the ScheduleME app using Figma. The next sections describe the different view of the high-fidelity prototype.

\section{Home Page}

The homepage of the schedule app is shown in Figure 3. The date is displayed at the top of the interface. Any message or quote the student would like to see on their home page can be added to the homepage as well. It can be a motivational quote or a student's favorite words.

\section{Notifications}

the recent updates are posted in the LMS. Consequently, the notification will be sent to the student through the app. So, students will get notifications about exams, meetings, assignments, presentations, etc. All the notifications the student has received can be viewed in the notification interface.

\section{Side Menu}

This is the side menu of the ScheduleME app (Figure 5). The student's name and student number are displayed at the top. There are four tabs on the side menu. They are my profile, today's schedule, deadlines, and modules. The sign out button is also at the bottom of the side menu. 
My Profile

The first tab in the side menu is "my profile" (Figure 6). All the details that are relevant to students such as registered year, semester, student email is displayed there.



Figure 3. Home Page

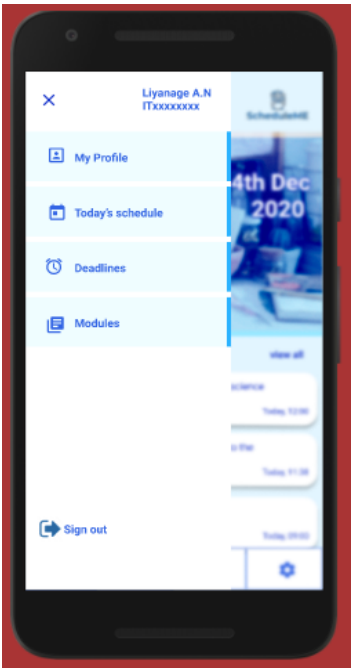

Figure 5. Side Menu



Figure 4. Notifications

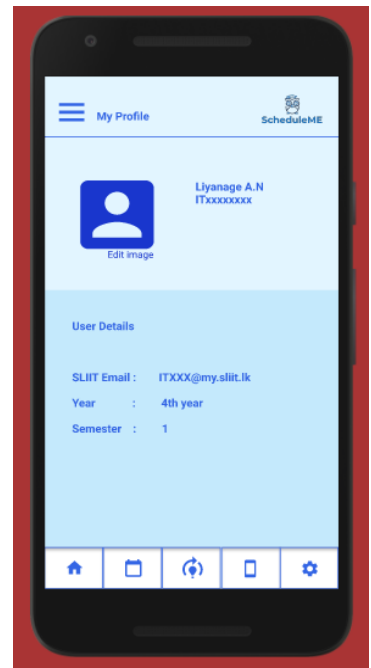

Figure 6. My Profile
Today's Schedule

Student's to-do list and completed tasks are listed here automatically with the scheduled time. So, the student can tick off the tasks they have completed. Also, a new task can be added manually by clicking the "Add" button.

\section{Upcoming Deadlines and Completed Deadlines.}

The deadline interface is divided into 2 different tabs. The first one is upcoming tasks (Figure 9). all the upcoming assignments, deadlines, quizzes, and their dates are listed here. And the time needed to allocate for each of those tasks is displayed as well. The student can edit that time as his/her preference.
In the completed deadlines tab (Figure 9), All the assignments and exams that are completed by the student are listed here, the student must update if he/she could submit the assignment before the deadline entering yes or no.

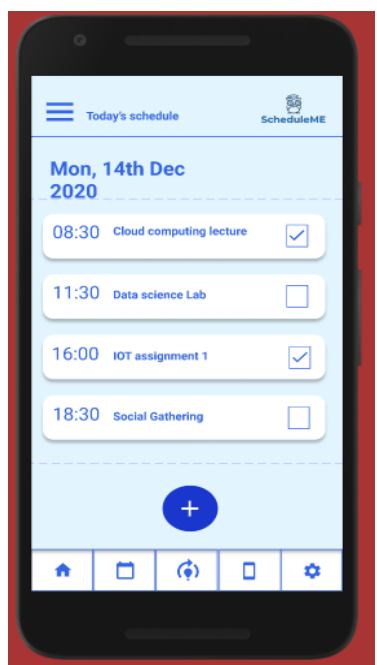

Figure 7. Schedule

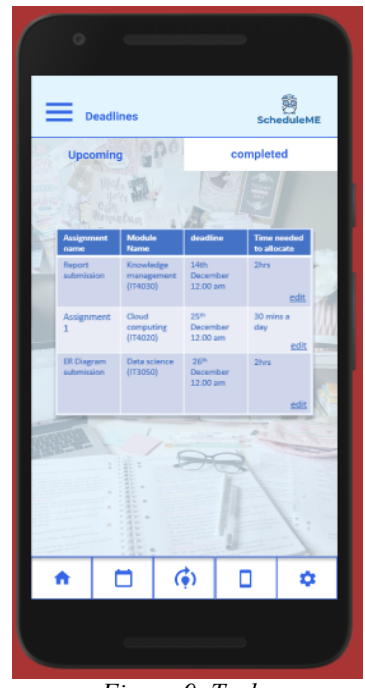

Figure 9. Tasks

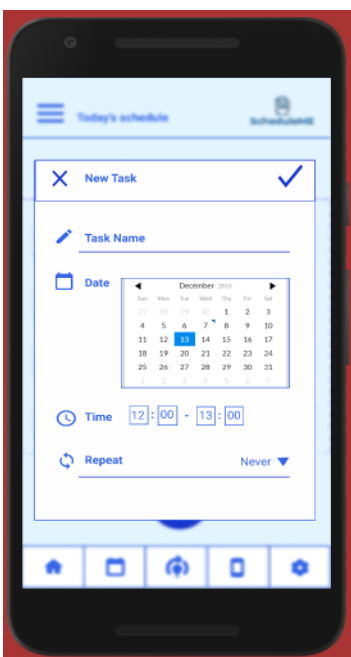

Figure 8. Add New Task

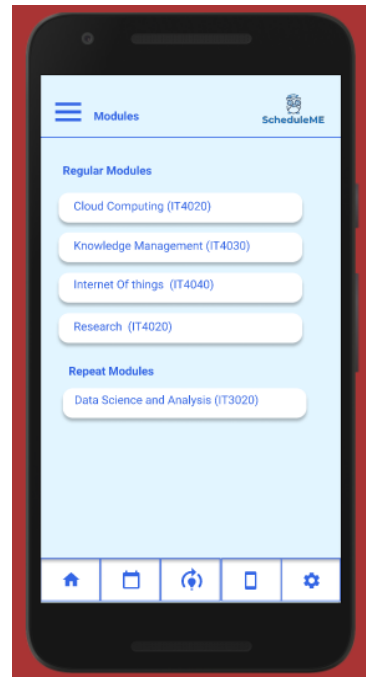

Figure 10. Modules
Modules

All the subjects the student has registered for the semester are displayed here. And the enrollment keys of those subjects are also displayed (Figure 10).

\section{Calendar}

The calendar is displayed as a list view according to the user's preference collected from low-fi prototypes feedback. All the schedules and students' daily timetable are displayed in the calendar interface. And the student can also add a task manually by clicking the add button. 
Progress

In Figure 12, the student can see the progress of the assignments that he/she is doing currently. It shows the completed percentage of each assignment and the deadlines of each assignment with time remaining. Then, the student can edit and update the progress by clicking the edit progress button.

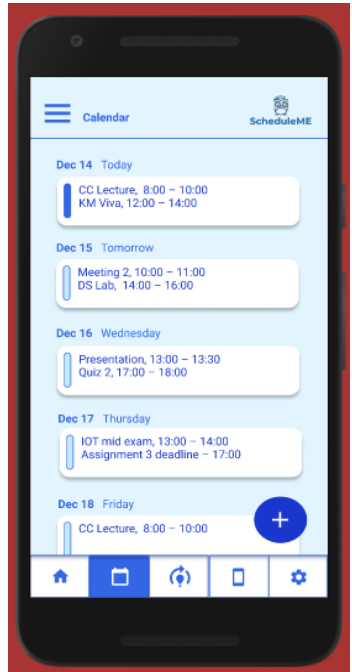

Figure 11. Calendar

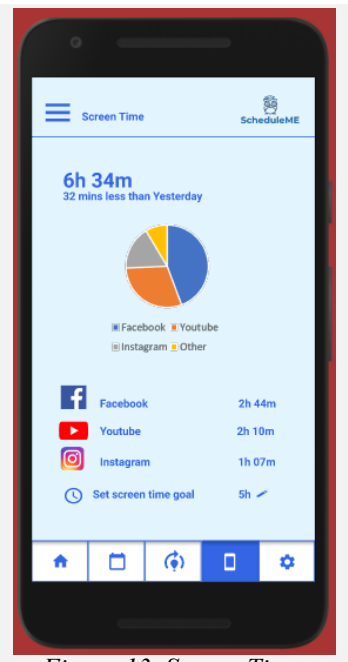

Figure 13. Screen Time



Figure 12. Progress

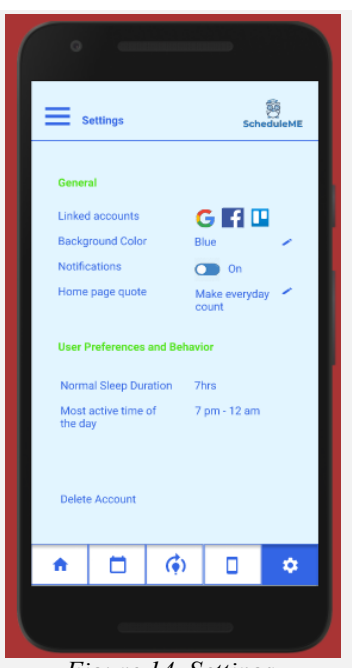

Figure 14. Settings
Screen Time

The student can view the times he/she spent on the phone in this interface. The most used apps and time spent on it can be viewed in this interface. And the student can set a screen time goal and try to achieve it. How much time he/she spent on all the social media, apps is displayed at the top of the UI.

\section{Settings}

Figure 14 shows the setting interface of the ScheduleME app. under "general settings", the student can manage their linked accounts such as Facebook, Google, etc., and the student can change the background color, homepage quote, etc. And under the "user preferences and behavior" settings, the student can edit his/her sleep duration and most active time of the day. According to those student inputs, task suggestions will be given.

\section{Testing}

The prototypes created in the previous stage are evaluated in this stage and the process iterated back to the design stage in most cases. Testing was done after high-fidelity prototypes were designed. It was performed by a small group of target end-users. They were asked to interact with the prototypes and rate their features and comment on them. Mainly focused on the user's ease to use the application, flexibility in handling controls, and the ability of the system to meet its objectives. A focus group was conducted to test the high-fidelity prototypes created in the last stage. 8 undergraduate students participated in the focus group.

The feature evaluation form was distributed among all the participants and collected feedback about the features of the mobile app.

- 1 star: I would not use it; It is hard to use.

- 3 stars: It can be useful sometime; It is neither hard nor easy to use.

- 5 stars: I will absolutely use it; It is easy to use

All the features in the mobile app were listed in the feature evaluation form. Each participant was asked to rate the features on how useful they are, and how easy it is to use them, giving 1-5 stars, after interacting with hi-fi prototypes. And they can add a comment on the feature as well. The features listed in the form were Notifications, Calendar, Today's schedule, Upcoming deadlines, completed deadlines, customized settings, Assignment progress, Manual task adding, Task prioritizing, Task Suggestions and Screen time. Overall, most of the ratings and comments were positive. But there were few ratings and comments that are negative, such as "if the app always suggests tasks, even in students' free time, it can be disturbing."

\section{DISCUSSION}

It is evident from the findings that previous research has not satisfactorily addressed the time management problems of university students. Further, it is noted that TMTs have not been designed specifically to fulfill the needs of university students who are reactive in nature. Existing TMTs are suitable for proactive and organized students, who possess the necessary perseverance and discipline to setup and configure the TMT and enter all the task details manually. This is not applicable to over half of students, who are reactive in nature and unorganized, as evident from the online survey and interview data that we gathered initially.

Further, this research sheds light on the common shortcomings of the existing TMTs via the questionnaires and interviews conducted in the empathize stage. They are: (a) "Having to enter all the details manually" (b) "Too much information or less information is shown in the app" (c) "Hard to prioritize the tasks" (d) "Does not make reminders" (e) "Less user-friendly" etc. The focus group conducted to investigate solutions to the user problems identified in the empathize and define stages. 
Prototypes of the mobile app were designed to provide solutions to the problems we found, according to the user needs and requirements we gathered. Finally, in the testing stage, highfidelity prototypes were evaluated by a group of representative end-users. Few modifications were made to the prototypes applying the feedback and comments we gathered during the testing stage.

\section{LIMITATIONS AND FUTURE WORK}

A limitation was the low sample size used for the prototype evaluation stage and the difficulty to interact with users face to face due to the COVID-19 pandemic situation at the time. Future research could have larger and more diverse sample populations for evaluating the prototypes. The next stage of this work will focus on developing the smart digital personal assistant named "ScheduleME" in a form of a mobile app according to the results and findings gathered throughout this research.

\section{CONCLUSION}

This work presents high-fidelity prototypes of an effective time management and task prioritizing system in a form of a mobile app for university students that is (a) straightforward to use and it does not require an unnecessary amount of time to learn and use (b) meets the needs of university students who are reactive in nature. A combination of User Centered Design and Design Thinking methodologies were conducted successfully with user input at each stage to develop high-fidelity prototypes. The research findings shed light on the time management problems faced by university students. Future researchers can use these findings to develop an effective and user-friendly digital personal assistant TMT for university students.

\section{REFERENCES}

[1] Baek, E., Cagiltay, K., Boling, E. and Frick, T. 2008. User-Centered Design and Development. Handbook of Research on Educational Communications and Technology.

[2] Abras, C., Maloney-Krichmar, D. and Preece, J. 2004. User-Centered Design. . Encyclopedia of HumanComputer Interaction

[3] Veryzer, R. and Borja de Mozota, B. 2005. The Impact of User-Oriented Design on New Product Development: An Examination of Fundamental Relationships*. Journal of Product Innovation Management. 22, 2 (2005), 128-143.

[4] Luchs, M. and Swan, K. 2011. Perspective: The Emergence of Product Design as a Field of Marketing Inquiry*. Journal of Product Innovation Management. 28, 3 (2011), 327-345.

[5] Liedtka, J. 2014. Perspective: Linking Design Thinking with Innovation Outcomes through Cognitive Bias Reduction. Journal of Product Innovation Management. 32, 6 (2014), 925-938.

[6] Questionnaires and surveys: 2021. https://www.slideshare.net/mJanekoh/questionnaires -and-surveys-32641698. Accessed: 2021- 08- 31.
[7] Darmayantie, A. 2020. Socio - Technical Perspective for Better Design Thinking Process in User Interaction Design Using Light-Weight Why Because Analysis (LWBA). International Journal of Scientific and Research Publications (IJSRP). 10, 7 (2020), 423-427.

[8] Asana vs Trello vs Slack vs Microsoft Teams vs Basecamp vs Todoist | We Rock Your Web: 2021. https://www.werockyourweb.com/management/proj ects/. Accessed: 2021- 08- 31

[9] Yue, P. and Beisler, A. 2014. Designing User-Centered Discovery and Access Services for Enhanced Virtual User Experience. The Serials Librarian. 66, 1-4 (2014), 268277.

[10] Wiggins, B. 2016. An Overview and Study on the Use of Games, Simulations, and Gamification in Higher Education. International Journal of Game-Based Learning. 6, 1 (2016), 18-29.

[11] Blandford, A. and Green, T. 2001. Group and Individual Time Management Tools: What You Get is Not What You Need. Personal and Ubiquitous Computing. 5, 4 (2001), 213-230.

[12] Dumitru, C. and Mittelstadt, A. 2020. What We Know and What We Do Not Know about Trust in Work Teams: A Systematic Literature Review. European Journal of Business and Management Research. 5, 3 (2020).

[13] Bartholomew, C. 2012. Time: An Empirical Analysis of Law Student Time Management Deficiencies. SSRN Electronic Journal. (2012).

[14] Kim, Y., Ho Jeon, J., Choe2, E., Lee, B. and Kim, K. 2016. TimeAware: Leveraging Framing Effects to Enhance Personal Productivity. CHI. (2016).

[15] Sandino, D., M, L. and Vélez, G. 2013. Design Thinking Methodology for the Design of Interactive Real-Time Applications. (2013)

[16] Trello: 2021. https://trello.com/en-US. Accessed: 202108- 31 .

[17] Todoist: The to do list to organize work \& life: 2021. https://todoist.com/home. Accessed: 2021- 08- 31.

[18] Google Calendar: 2021. https://calendar.google.com/. Accessed: 2021- 08- 31

[19] Czerwinski, M., Horvitz, E. and Wilhite, S. 2004. A diary study of task switching and interruptions. Conference on Human Factors in Computing Systems (New York, NY, United States, 2004), 175-182.

[20] Paul, C. L., Komlodi, A., \& Lutters, W. 2015. Interruptive notifications in support of task management. International Journal of Human-Computer Studies, 79, 20-34.

[21] Franssila, H. 2019. Work fragmentation, task management practices and productivity in individual knowledge work. In International Conference on Human-Computer Interaction (pp. 29-38). Springer, Cham.

[22] Franssila, H., Okkonen, J., \& Savolainen, R. 2014. Email intensity, productivity and control in the knowledge worker's performance on the desktop. In Proceedings of the 18th International Academic MindTrek Conference: Media Business, Management, Content \& Services (pp. 19-22). 